\title{
Dynamical Behavior of Logistic Maps
}

\author{
Umme Ruman \\ Department of Mathematics \\ Atish Dipankar University of \\ Science \& Technology \\ Bangladesh.
}

\author{
Rebeka Tanij Tania \\ Department of Computer \\ Science \& Engineering \\ Atish Dipankar University of \\ Science \& Technology \\ Bangladesh.
}

\author{
Muhammad Abdus Sattar \\ Titu \\ Department of Mathematics \\ Atish Dipankar University of \\ Science \& Technology \\ Bangladesh.
}

\begin{abstract}
In this paper, we study basic dynamical facts for logistic growth models in population dynamics and its dynamical behavior. Different logistic growth curves have been developed and more general biological logistic growth curve are studied. We also discuss the concept of bifurcation in the context of logistic growth models.
\end{abstract}

\section{General Terms}

Bifurcation, logistic maps, Algorithms.

\section{Keywords}

Dynamical system, logistic behavior.

\section{INTRODUCTION}

We ask that The real quadratic logistic growth family of curves $f_{a}(x)=\operatorname{ax}(1-x)$,

was recognized as a very interesting and representative model of real dynamical systems. The simplest logistic growth maps are quadratic polynomial, which depends on a single parameter a, chaotic dynamical behavior [2] also appears in the system. The simple exponential growth model provide interesting biological model.

\section{DYNAMICAL SYSTEMS}

The Dynamical system is the time evaluation of any systems. $(X, G, \phi)$ following conditions:

a) $X$ be a non-empty set.

b) $G$ be a group or semi-group.

c) $\phi: X \times G \rightarrow X$ satisfying

$$
\begin{aligned}
& \phi\left(\phi\left(x, g_{1}\right), g_{2}\right)=\phi\left(x, g_{1} * g_{2}\right), \forall g_{1}, g_{2} \in X \\
& \text { and } \phi(x, \circ)=x
\end{aligned}
$$

When a scientist confronts dynamical systems, the question that she or he asks can i predict what will happen in the future, can i predict how this motion will evolve in time? If you look at some of the examples giving of dynamical systems, it is clear that some of them are predictable.
On the other hand, the weather or the stock market, those are examples of dynamical systems that seem to be unpredictable. The question now, is why are they so unpredictable?

For example to predict the weather, you would have to know all elements of the weather around the globe instantaneously. You would have to know the barometric pressure, the wind speed and direction everywhere in the globe in order to predict what the weather will be like a week.

rs capitalized and 6-points of white space above the subsubsection head.

\section{LOGISTIC GROWTH MODEL}

To overcome this deficiency in the mathematical model, Verhulst in 1838 proposed an infinite growth rate

$$
r=r(p)=a-b p \quad(a>0, b>0, b<a)
$$

which decreases linearly as population size increases. The resulting growth mode

$p\left(t_{0}\right)=p_{0}>0$ where $p_{0}$ denotes the initial population size at time $t_{0}$

$$
p^{\prime}(t)=\operatorname{ap}\left(1-\frac{p}{k}\right)
$$

where $k=\frac{a}{b}$ (say)

where $k$ is carrying capacity of the environment. The solution of (2) is given by

$$
p(t)=\frac{k}{1+\left(\frac{k}{p_{0}}-1\right) e^{-a t}}
$$

Behavior of this Solution

(i) $\lim _{t \rightarrow \infty} p(t)=k$, the population will ultimately reach its carrying capacity.

(ii) The relative growth rate declines linearly with increasing population size and reaches its zero minimum $p=k$. 
For $a>0$, the growth curve, is asymptotic to the carrying capacity. If $a=0$, the population remains constant at the initial value of $p_{0}$. The population size $p$ verses time t for the logistic from the solution of (2) for the following parameter values:

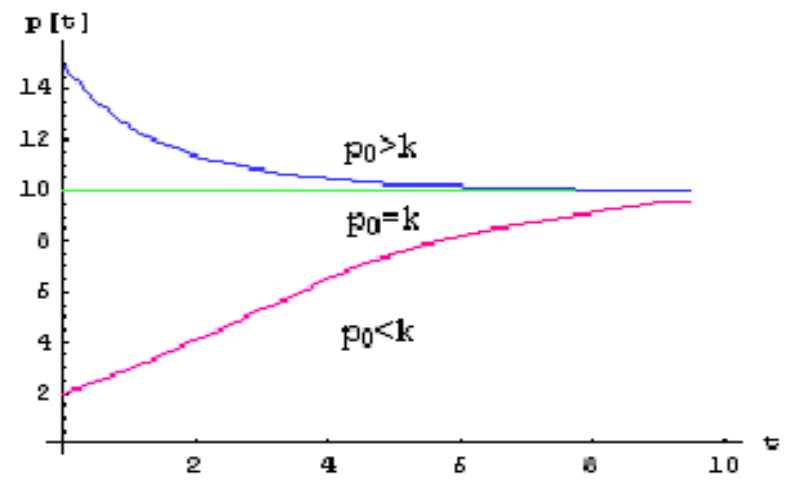

Figure 3.1: Logistic growth model with (i)

$$
\begin{aligned}
& a=0.5, p_{0}=10, k=10 \\
& \text { (ii) } a=0.5, p_{0}=15, k=10 \\
& \text { (iii) } a=0.5, p_{0}=2, k=10
\end{aligned}
$$

So we conclude that the time period before the population reaches half of its limiting value is a period of accelerated growth and after this point, the rate of growth decreases and in course of it reaches zero, this being a period of diminishing growth. The above observations are depicted in the Figure 3.1.

\section{DYNAMICAL BEHAVIOR OF LOGISTIC MAP}

Consider $f_{a}(x)=\operatorname{ax}(1-x), 0 \leq a \leq 4$ First we find out the fixed point of $f_{a}(x)$.

Here, $x=0, \quad x=p_{a}:=1-\frac{1}{a}$, are fixed points of $\mathrm{f}$, where $0 \leq p_{a}<1$.

Note that changing the parameter a changes the rate at which the asymptotic values $a_{\infty}$ is reached.

Here $f_{a}^{\prime}(x)=a-2 a x$. Therefore, $f_{a}(x)$ has only one fixed point $x=0$ iff $a \leq 1$. For small growth rate $a<1$ (say, $a=1 / 2$ ), the population always dies out no matter what the initial condition is.

In this case $a>1, f_{a}$ has two fixed points $x=0$ and $x=(a-1) / a$. Here, $\max f(x)=f(1 / 2)=\frac{a}{4}$.

So, $\quad f:[0,1] \rightarrow[0,1]$ if and only if $a \leq 4$. Here $f_{a}^{\prime}(0)=a$ and $f_{a}^{\prime}\left(p_{a}\right)=2-a$. Hence 0 is repelling fixed point for $\mathrm{a}>1$ and attracting fixed point for $0<a<1$. Also $p_{a}$ is attracting for $1<a<3$ and is repelling if $a>3$ or a $<1$. Here, $p_{a}$ is non-hyperbolic or neutral fixed point when $a=3$ is non-hyperbolic or neutral fixed point when $a=3$.

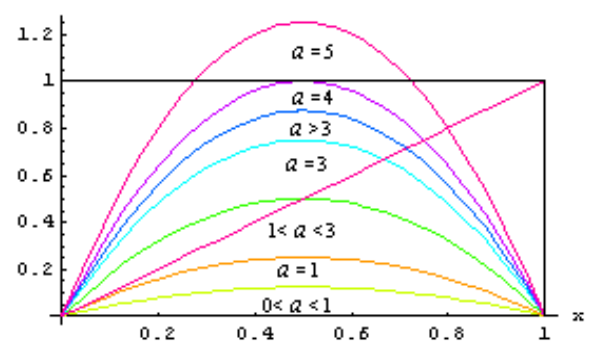

Figure 4.1: Logistic map for different values of $a$.

Now Periodic Points of Period "2"

For periodic points with prime period 2, we have to consider $f^{2}(x)=x$.

For $a>3, a^{2} x^{2}-a(a+1) x+a+1=0$ has two real solution say $q_{1,2}$ where

$$
q_{1,2}=\frac{a+1 \pm \sqrt{(a+1)(a-3)}}{2 a} .
$$

We have $f\left(q_{1}\right)=q_{2}$ and $f\left(q_{2}\right)=q_{1}$, that is,

$q_{1}, q_{2}$ are periodic points with prime period 2 for $a>3$.

Thus $q_{1}, q_{2}$ are attracting periodic points with prime period 2 if and only if $\left|\left(f_{a}^{2}\right)^{\prime}\left(q_{1}\right)\right|=\left|4+2 a-a^{2}\right|<1$, that is, $3<a<1+\sqrt{6} \approx 3.45$.

For $a=4$, the map is chaotic on [0,1] For $a>2+\sqrt{5}$, there is a Cantor set in $[0,1]$ on which $f_{a}$ is chaotic when $a>2+\sqrt{5}$.

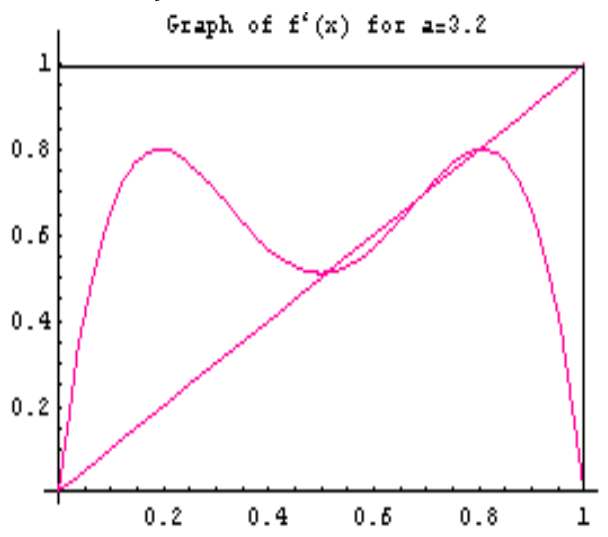



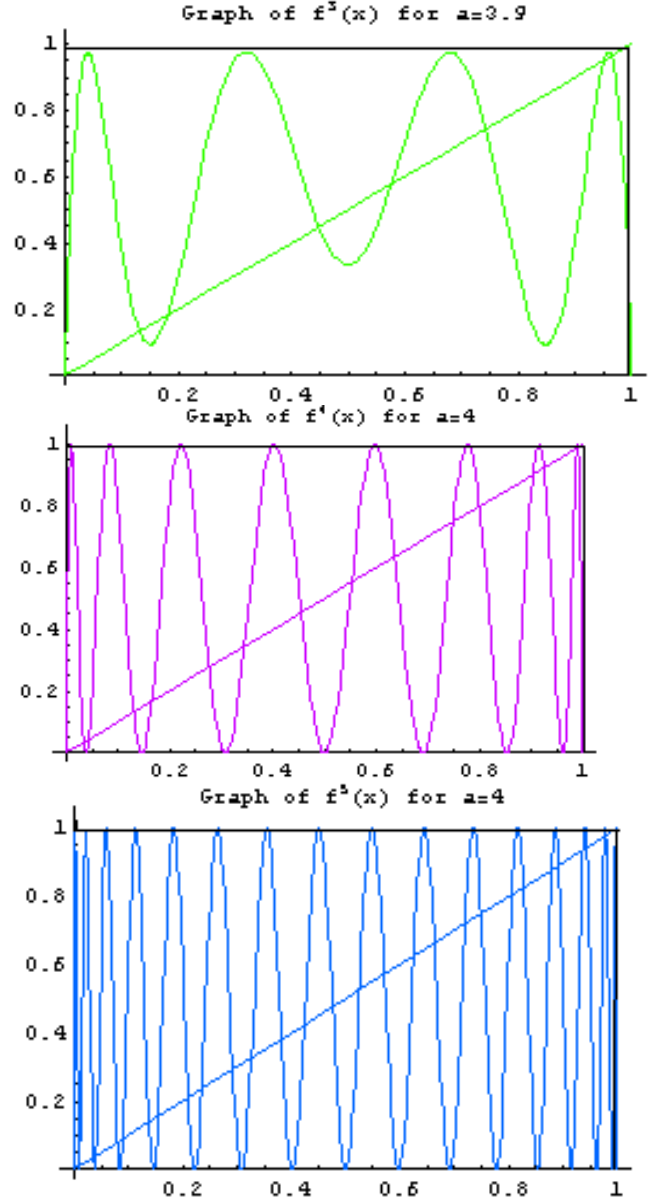

Figure 4.2: Logistic map for period "2", "3", "4", "5"

\section{BIFURCATION OF THE LOGISTIC}

\section{MAP}

For $a>3$, changes to repelling and a 2 cycle is born.

The system exhibits some interesting phenomena [4], which cannot be observed from the continuous logistic system.

For $3<a<1+\sqrt{6} \approx 3.45$, the two cycle is stable the population may oscillate between two values forever.

For $a=1+\sqrt{6} \approx 3.544090$, the 2 cycle becomes unstable and a stable 4 cycle is born then the population may oscillate between four values forever.

For $1+\sqrt{6}<a<1+2 \sqrt{2} \approx 3.544090$, the 4 cycle becomes unstable and a stable 8 cycle is born, when $a$ is slightly bigger than 3.54 then 8 than 16,32 etc.

In general, a stable $2^{k}$ cycle is born at $a_{k}$ and becomes unstable at $a_{k+1}$, where

$$
\begin{gathered}
a_{1}=3 \\
a_{2}=3.449 \ldots \\
a_{3}=3.54409 \ldots \\
a_{4}=3.5644 \ldots \\
a_{5}=3.568759 \ldots \\
a_{6}=3.569692 \ldots \\
a_{7}=3.5698 \ldots \\
a_{8}=3.569946 \ldots
\end{gathered}
$$

It should be clear that as $n$ goes large these values are approaching a limit

$$
a_{\infty}=\lim _{n \rightarrow \infty} a_{n}=3.569946 \ldots
$$

It means that an $\infty$ cycle for the value of $a$. Note that the successive bifurcations come faster and faster. The convergence is essentially geometric; the limit of large $n$, the distance between successive transitions shrinks by a constant factor

$\delta=\lim _{n \rightarrow \infty} \frac{a_{n}-a_{n-1}}{a_{n+1}-a_{n}}=4.6692016091$

It should be noted that $a_{\infty}<4$, which means that all iterates are confined. When $a>a_{\infty}$, behavior turns out to be chaos. For $a_{c}<a<4$. It is difficult to analysis this case. The sequence $\left\{x_{n}\right\}$ never settles down to a fixed point or a periodic point, instead the long term behavior is a periodic.

The points that are plotted will (within the resolution of the picture) approximate either fixed or periodic sinks or other attracting sets. This figure is called the bifurcation diagram and shows the birth, evolution, and death of attracting sets. The term "bifurcation" refers to significant changes in the set of fixed or periodic points or other sets of dynamic interest.

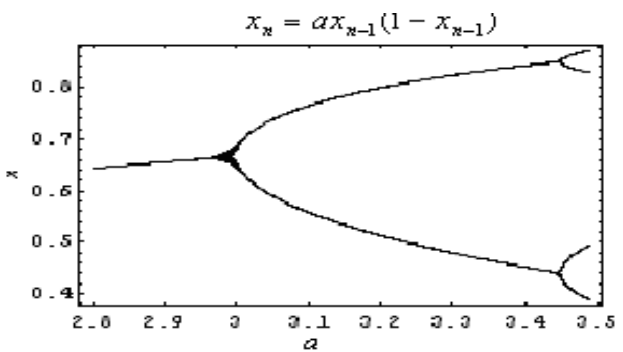


Figure 5.1 Bifurcation diagram of

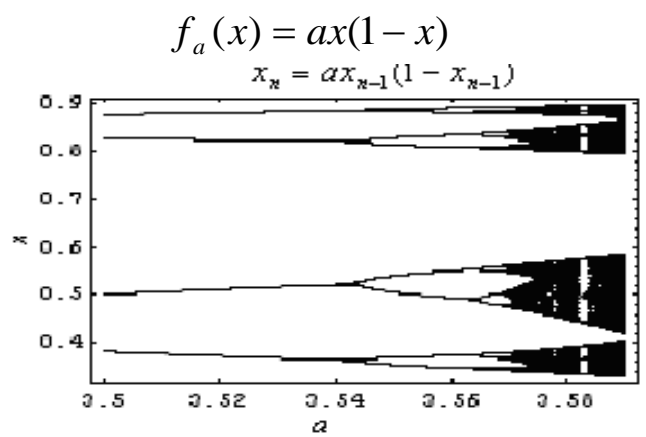

Figure 5.2: Magnification of the logistic bifurcation diagram for $3.5 \leq a \leq 3.58$.

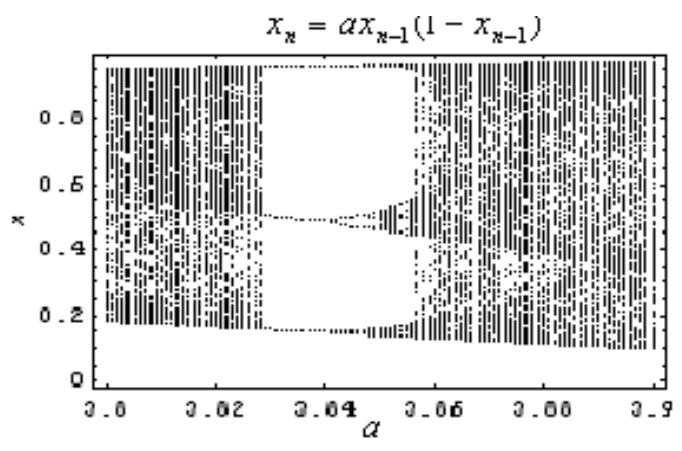

Figure 5.3: Magnification of the logistic bifurcation diagram for $3.8 \leq a \leq 3.9$

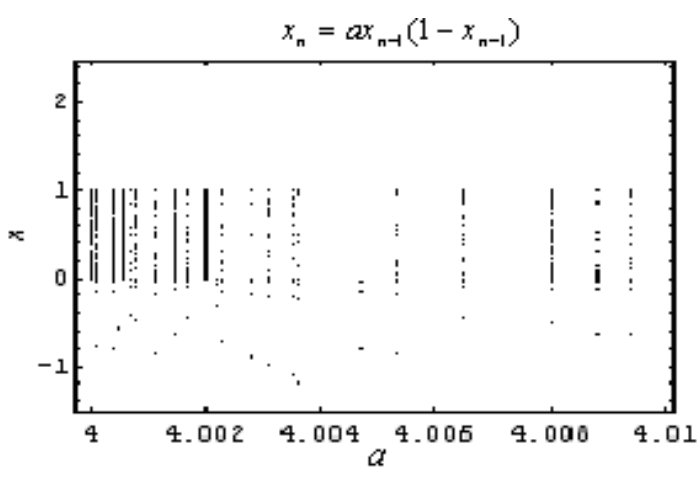

Figure 5.4: Magnification of the logistic bifurcation

diagram for $4 \leq a \leq 4.01$

\section{CONCLUSION}

The purpose of study in this paper is to investigate the dynamical behavior of one dimensional non-linear logistic maps which arises from population dynamics.

The real quadratic logistic growth family of curves $f_{a}(x)=\operatorname{ax}(1-x)$ was recognized as very interesting and representative model of real dynamical systems. For $a \leq 1, f_{a}(x)$ has only one fixed point and $a>1, f_{a}(x)$ has two fixed points. The fixed point 0 is a repelling fixed point for $a>1$ and attracting fixed point if $0<a<1$ and neutral fixed point for $a=1$. for $a \geq 1+2 \sqrt{2}$, there exists periodic points with prime period 3 .

For $a>3$, changes to repelling and a 2 cycle is born. The system exhibits some interesting phenomena, which cannot be observed from the continuous logistic system.

For $3<a<1+\sqrt{6} \approx 3.45$, the two cycle is stable the population may oscillate between two values forever. For $a=1+\sqrt{6} \approx 3.544090$, the 2 cycle becomes unstable and a stable 4 cycle is born then the population may oscillate between four values forever.

For $1+\sqrt{6}<a<1+2 \sqrt{2} \approx 3.544090$, the 4 cycle becomes unstable and a stable 8 cycle is born, when $a$ is slightly bigger than 3.54 then 8 than 16,32 etc. In general stable $2^{k}$ cycle is born at $a_{k}$ and becomes unstable at $a_{k+1}$.

We show that for $a>4$ the set $\Lambda=\left\{x \mid f_{a}^{n}(x) \in[0,1], \forall n\right\}$ is a Cantor set [1] and the function $f_{a}: \Lambda \rightarrow \Lambda$ is chaotic

\section{ACKNOWLEDGMENTS}

Thanks all who have participated and help to complete this research works and also thanks senior faculty members of our department.

\section{REFERENCES}

[1] Devaney, R.L., A First Course in Chaotic Dynamical Systems, Perseus Press, 1993.

[2] Devaney, R.L., An Introduction to Chaotic Dynamical Systems, Westview Press, 2003.

[3] Holmgren R.A, A First Course in Discrete Dynamical Systems, spring-verlag, 1996.

[4] Robert M.May, Simple Mathematical Models with very Complicated Dynamics,Nature 261, 459-467, 1976.

[5] M. Martelli, On the Definition of Chaos.

[6] Wiley-Interscience, Introduction to Discrete Dynamical Systems and Chaos ,1999.

[7] Li, T.-y., and Yorke J., Period Three Implies Chaos .American Mathematical Monthly 82 (1975), 985-992. 